\title{
BIOINFORMATION
}

Discovery at the interface of physical and hiological sciences

\section{An insight into the lignin peroxidase of Macrophomina phaseolina}

\author{
Mohammed Touaha Akbar, Abdul Musaweer Habib, Dil Umme Salma Chowdhury, Md Iqbal \\ Kaiser Bhuiyan, Kazi Md Golam Mostafa, Sobuj Mondol \& Ivan MHAI Mosleh*
}

Department of Genetic Engineering and Biotechnology, Faculty of Biological Sciences, University of Chittagong, Chittagong - 4331, Bangladesh; Ivan MHAI Mosleh - Email: ivanahmed96@yahoo.com; *Corresponding author

Received July 15, 2013; Accepted July 16, 2013; Published August 07, 2013

\begin{abstract}
:
Macrophomina phaseolina is one of the deadliest necrotrophic fungal pathogens that infect more than 500 plant species including major food, fiber, and oil crops all throughout the globe. It secretes a cocktail of ligninolytic enzymes along with other hydrolytic enzymes for degrading the woody lignocellulosic plant cell wall and penetrating into the host tissue. Among them, lignin peroxidase has been reported only in Phanerochaete chrysosporium so far. But interestingly, a recent study has revealed a second occurrence of lignin peroxidase in M. phaseolina. However, lignin peroxidases are of much significance biotechnologically because of their potential applications in bio-remedial waste treatment and in catalyzing difficult chemical transformations. Besides, this enzyme also possesses agricultural and environmental importance on account of their role in lignin biodegradation. In the present work, different properties of the lignin peroxidase of M. phaseolina along with predicting the 3-D structure and its active sites were investigated by the use of various computational tools. The data from this study will pave the way for more detailed exploration of this enzyme in wet lab and thereby facilitating the strategies to be designed against such deadly weapons of Macrophomina phaseolina. Furthermore, the insight of such a ligninolytic enzyme will contribute to the assessment of its potentiality as a bioremediation tool.
\end{abstract}

Key words: Macrophomina phaseolina, Ligin peroxidase, lignin.

\section{Background:}

Plant biomass is primarily consists of cellulose, lignin and hemicellulose. Lignin is the most abundant natural polymer which makes 15 to $30 \%$ of the woody cell walls of gymnosperms (softwood) and angiosperms (hardwood). An encrusting matrix is formed by lignin around the cellulose, the most abundant natural polymer. As this matrix significantly prevents the microbial de-polymerization of cellulose, lignin degradation is a crucial step in the global carbon cycle. Furthermore, the presence of this imperturbable polymer is responsible for the inefficient utilization of cellulose in a wide range of industrial processes [1].

Lignin peroxidase (Lip) is a monomeric haemoglycoprotein peroxidase secreted by the white wood-rot fungus Phanerochaete chrysosporium. Lignin peroxidases are of much interest biotechnologically because of their potential applications in bioremedial waste treatment and in catalyzing difficult chemical transformations. Due to their role in lignin biodegradation they are also of agricultural and environmental importance [2].

A recent study revealed the second occurrence of lignin peroxidase in Macrophomina phaseolina, which is one of the most devastating necrotropic fungal pathogens that infect more than 500 plant species throughout the world. It invades the lignocellulosic barrier in fibre crop jute with the help of various ligninolytic and cellulolytic enzymes. The lignin depolymerizing enzymes are laccases, lignin peroxidases, galactose oxidases, and chloroperoxidases, haloperoxidases, and heme peroxidases. Among them, lignin peroxidase is the one, which is main concern of our study. Following invasion, M. phaseolina can grow rapidly in infected jute plant and 
produces a large amount of sclerotia which plugs the vessels, and then causes wilting of the plant [3].

So, identifying different properties of this enzyme from $M$. phaseolina along with predicting the 3-D structure and its active sites could be helpful in designing strategies against this catastrophic fungus. Moreover, pollutions owing to olive mill wastewaters (OMW) is creating a serious ecological problem in the Mediterranean area, particularly in Italy, Spain, Greece, and Tunisia, which are the main producers of olive oil. These countries produce more than $3 \times 10^{7} \mathrm{~m}^{3}$ of OMW per year. Due to high concentration of aromatic compounds, this effluent is black \& highly noxious [4]. This wastewater includes the black high-molecular-weight polyphenols such as tannins, anthocyanins, and catechins [5]. The phytotoxic and antibacterial effects of the OMW have been attributed to the phenolic content of OMW [6]. Conventional bacterial water treatment processes are relatively ineffective at removing these pollutants. OMW must be diluted more than 10 -fold prior to treatment with aerobic activated sludge or by anaerobic digestion. Hence, it requires more effective treatment.

The irregular and recalcitrant nature of lignin and the fact that the substructures contained in lignin are also found in primary pollutants such as phenols, anisoles, biphenyls, and diarylethers led researchers to postulate that the nonspecific ligninolytic enzyme produced by white rot fungi might be able to degrade such persistent aromatic pollutants. Moreover, a wide variety of phenolic compounds and environmentally persistent xenobiotics and chlorinated hydrocarbons, including DDT [1,1,1-trichloro-2,2-bis(4-chlorophenyl)ethane], TNT (2,4,6trinitrotoluene), alkyl halide insecticides, chloroanilines, polychlorinated phenols, and polychlorinated biphenyls are degradable by $P$. chrysosporium Thus, characterization of lignin peroxidase enzyme from $M$. phaseolina will be an added benefit in accordance with P. chrysosporium in the field of bioremedial waste treatment [7].

Until now, purification from the native fungus (Phanerochaete chrysosporium) has been the only method of producing the active enzyme [8]. Heterologous expression in E. coli genes, encoding the mature sequence of Lip from $P$. chrysosporium has been done successfully. Moreover, the first successful homologous expression of rLiPH8 in P. chrysosporium and the characterization of the recombinant enzyme has also been reported as well as enlightened the fact that increased expression of Lignin peroxidase in M. phaseolina can also be achieved [9]. In this present study, several features of lignin peroxidase of $M$. phasaeolina have been revealed using different web based tools.

\section{Methodology:}

Several characteristics of $M$. phaseolina have been identified along with its 3D structure and active site prediction by using different databases and software's maintaining as shown (Figure 1).

\section{Sequence retrieval and physicochemical characterization}

At the beginning, protein sequence of lignin peroxidase of $M$. phaseolina was retrieved in FASTA format from NCBI [10] and then physicochemical properties of the enzyme were obtained by using ProtParam [11].

\section{Sequence retrieval and physiochemical characterization}

2. Demonstration of proteolytic cleavage sites.

1

3. Conserved domain prediction.

$+$

4. 3D structure prediction.

5. Validation of model.

1

6. Active site prediction.

Figure 1: Methodolgy involved in characterisation of $M$. phaseolina.

\section{Identification of proteolytic cleavage site}

Proteolytic cleavage site was identified by using a web based tool, peptide cutter [12] which predicts protease cleavage sites and sites cleaved by chemicals in a given protein sequence.

\section{Identification of conserved domain}

To search the conserved domains present in the Lignin Peroxidase of Macrophomina phaseolina, we utilized Conserved Domain Search tool which can be found in NCBI [13]. The sequence can be added to this tool by the use of accession number, gi, or sequence in FASTA format. In this study, we added sequence in FASTA format.

\section{Prediction of three-dimensional structure}

To predict the three dimensional structure of Lignin peroxidase, the most popular tool, Phyre2 (Protein Homology/Analogy Recognition Engine) [14] an online protein fold recognition server, was used in this study. The retrieved amino acid sequence in FASTA format was used as input data in Phyre2. In this respect, the intensive mode of protein modeling was selected in order to get an accurate model.

\section{Validation of lignin peroxidase model}

To check the accuracy of the predicted three dimensional structure, PDBsum was used. PDBsum is a database that provides an overview of the protein structures stored in the Protein Data Bank for the protein structures that have already been generated. PDBsum has a page for each structure denoted by a specific PDB id. But for lignin peroxidase, there is no PDBsum page. So a customized page for lignin peroxidase was generated by using PDBsum 'generate' option [15]. In this case the PDB file obtained by using Phyre2 server for lignin peroxidase was used as input data.The PDBsum generated result was further subjected to documentation analysis by PROCHECK.To obtain an idea about the protein chains and 
their steriochemical quality, like Ramachandran plot quality, peptide bond planarity, Bad non-bonded interactions, main chain hydrogen bond energy, $\mathrm{C}$ alpha chirality and over-all $\mathrm{G}$ factor and the side chain parameters like standard deviations of chi1 gauche minus [16] PROCHECK analysis was done.

\section{Active site prediction}

To predict the active site of the sequence of interest a web based tool named CastP was used [17]. The weighted Delaunay triangulation and the alpha complex for shape measurements are used by CastP which provides identification and measurements of surface accessible pockets as well as interior inaccessible cavities, for proteins and other molecules. It assesses analytically the volume as well as the area of each cavity and pocket, both in molecular surface (MS, Connolly's surface) and solvent accessible surface (SA, Richards' surface). It also measures the number of mouth openings, circumference of mouth lips, and area of the openings, in both SA and MS surfaces for each pocket [18].

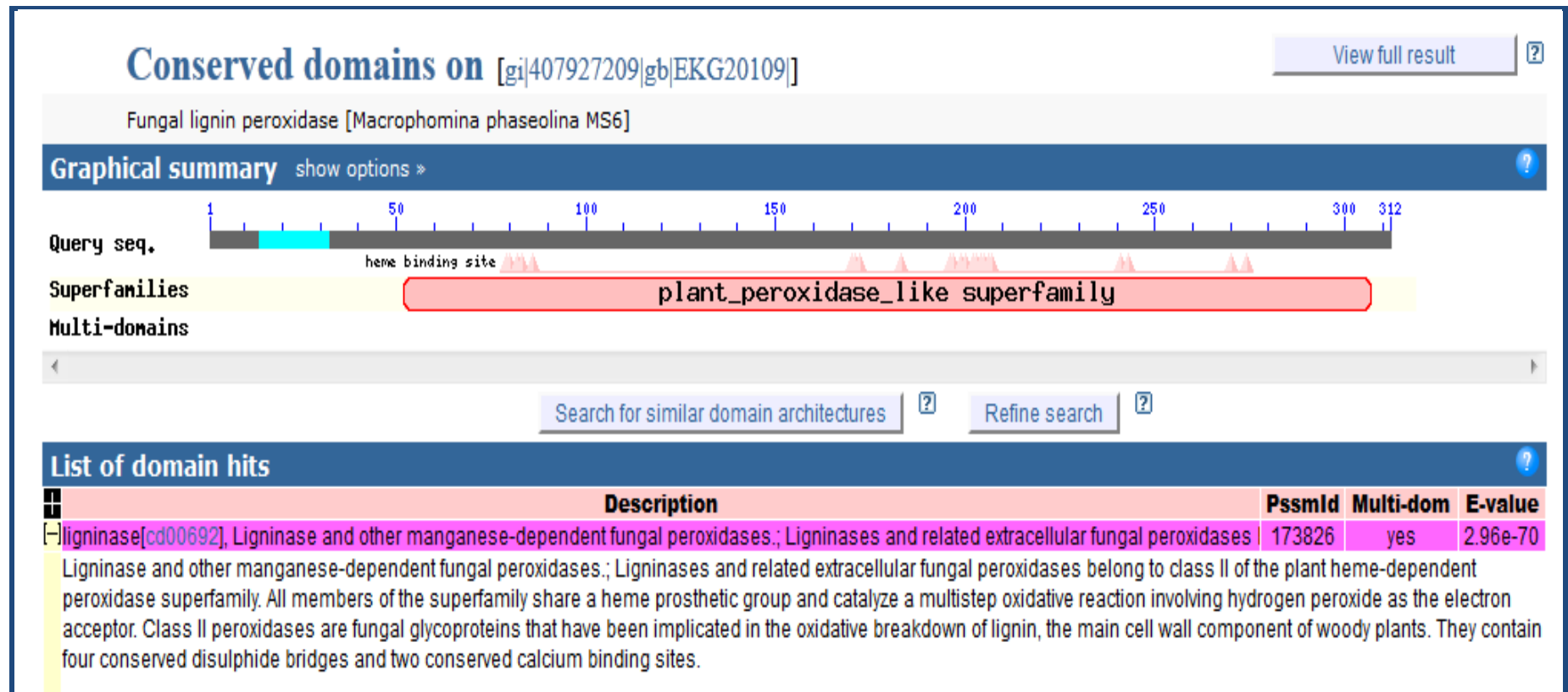

Figure 2: Conserved domain over lignin peroxidase

\section{Results \& Discussion:}

\section{Physicochemical characterization}

Different physicochemical properties of Lignin peroxidase has been characterized.Computation of Isoelectric point (theoretical pI) and molecular weight $(\mathrm{Mw})$ of an amino acid sequence is important because these data dictate the approximate condition of a 2D-gel in which a protein of interest can be detected [19]. Lignin peroxidase is moderately acidic (5.22) in nature. The instability index (II) for the respective enzyme is computed to be 29.87 which classifies the protein as stable. A protein whose instability index is smaller than 40 is predicted as stable; a value above 40 predicts that the protein may be unstable [20].

The half-life is the time which is taken by half of the amount of protein in a cell to disappear after its synthesis in the cell. The half-life is given for three organisms (human, yeast, and E. coli). The estimated half-life is: 30 hours (mammalian reticulocytes, in vitro; $>20$ hours (yeast, in vivo); $>10$ hours (Escherichia coli, in vivo) [20].

The relative volume of a protein occupied by its aliphatic side chains is termed as Aliphatic index (AI). Aliphatic index plays role in protein thermal stability. The higher the Aliphatic index of proteins, the more thermally stable the proteins. Aliphatic amino acids also are hydrophobic in nature [20]. The aliphatic index of lignin peroxidase is 86.44 which indicated this enzyme is thermally stable as well as it have high amount of hydrophobic amino acids. The protein is hydrophobic (due to positive GRAVY value: 0.022 ).

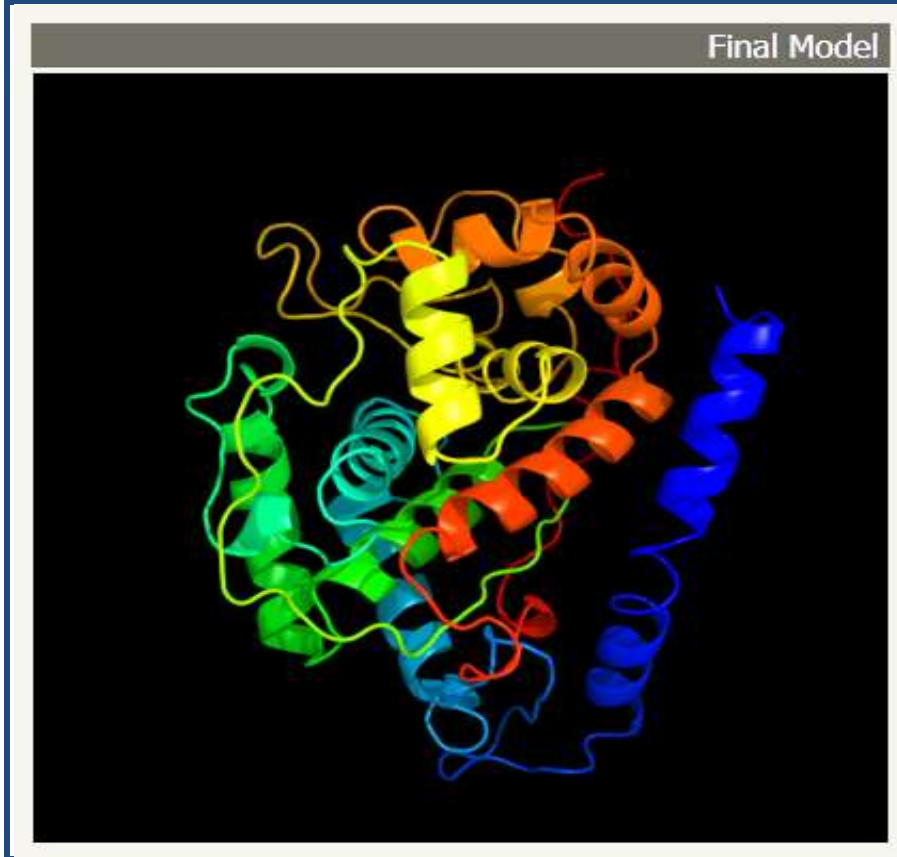

Figure 3: The final 3D structure of lignin peroxidase model from Macrophomina phaseolina. The a-helices and $\beta$-sheets are 
represented by redhelices and yellow ribbons respectively. This model was conducted by Phyre2 MODELLER program.

\section{Identification of proteolytic cleavage site}

Protease digestion can be useful if one wants to carry out experiments on a portion of a protein, separate the domains in a protein, remove a tag protein when expressing a fusion protein, or make sure that the protein under investigation is not sensitive to endogenous proteases. One or several reagents can be selected from a list of (currently) 33 proteases and chemicals [20]. The proteolytic cleavage sites found in lignin peroxidase enzyme of Macrophomina phaseolina is given in Table $\mathbf{1}$ (see the supplementary).

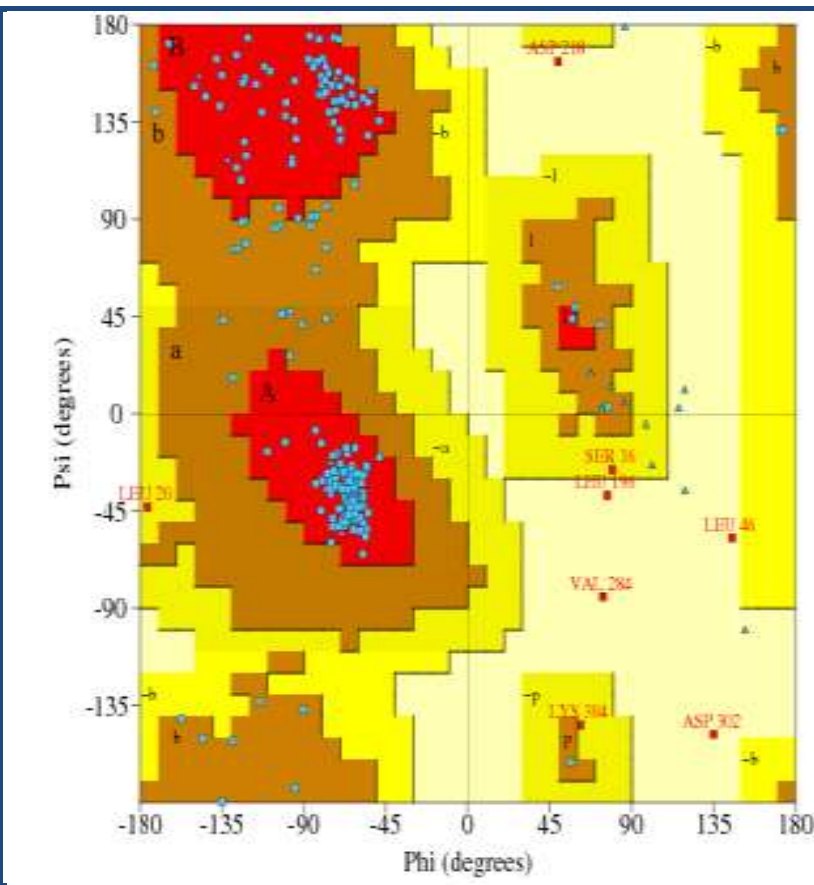

Figure 4: Ramachandran's Map of lignin peroxidase model from Macrophomina phaseolina. The Plot calculation was done with PROCHECK program

\section{Identification of conserved domain}

The fundamental units of protein structure, function, and evolution are known as protein domain. As a result, domain prediction is critical step in the annotation of protein sequences [21]. Domain predictions are also the starting point for a range of more sophisticated analyses, including comparative genomics of domain families in diverse organisms studies of the evolution of protein and domain structure and function, prediction of protein-protein interactions and identification of complex evolutionary relationships [22].

The conserved domain of lignin peroxidase lies between 30-300 amino acids of the enzyme (Figure 2). Within the conserved domain, there are several heme-binding sites. This enzyme belongs to a group of peroxidases or a superfamily named plant peroxidase-like superfamily. The enzyme,lignin peroxidase contain a heme prosthetic group (ferriprotoporphyrin IX), which catalyzes a multistep oxidative reaction involving hydrogen peroxide as the electron acceptor.

\section{3-D sructure determination}

Three-dimensional (3D) protein structures provide valuable insights into the molecular basis of protein function, allowing an effective design of experiments, such as site-directed mutagenesis, studies of disease-related mutations or the structure based design of specific inhibitors [23]. Therefore, the high resolution 3D structure of a protein is the key to the understanding and manipulating of its biochemical and cellular functions [24].

The theoretical structure (Figure 3) of lignin peroxidase from $M$ phaseolina is generated using Phyre2. Greater than $90 \%$ confidence match was obtained, which is a high confidence match. This implies that, overall fold of the model was almost certainly correct and the central core of the model was tend to be accurate [25].

\section{Validation of Lignin peroxidase Model}

Accuracy of the protein model was measured by PROCHECK. Parameter comparisons of these proteins were made with wellrefined structures that have similar resolution. Ramachandran plot and Ramachandran plot statistics is shown in (Figure 4) and Table 2 (see the supplementary). Thus, analysis of PROCHECK reveals that all residues are within the limits of the Ramachandran plot and hence it can be considered as a good model.

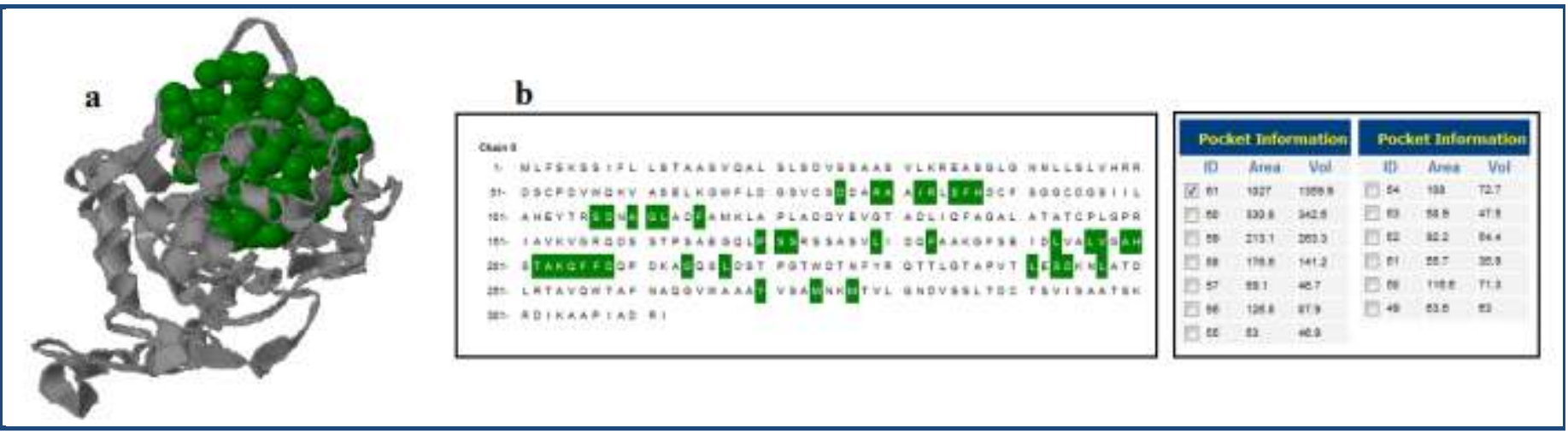

Figure 5: a) Active site information by CASTp. Green color shows the active site position from 76 to 287 with the $\beta$-sheet in between them and 3D representation of the best active site of lignin peroxidase generated by using CASTp; $\mathbf{b}$ ) The table shows the area and the volume for different active sites of lignin pefroxidase and the best active site remains in an area of 1027 and a volume of 1359.9 amino acid. 


\section{Active site prediction}

The identification and characterization of functional sites on proteins has increasingly become an area of interest. Because analysis of the active site residues for the binding of ligands provides insight towards the design of inhibitors of an enzyme [26]. In this study, we have also reported the best active site area of the experimental enzyme as well as the number of amino acid involved in it. (Figure $5 a \mathbf{a} \mathbf{5 b}$ ).

\section{Conclusion:}

Lignin peroxidase of $M$. phaseolina, an enzyme having undoubted biotechnological prospect, was terra incognita. This work has unearthed its several crucial aspects, on the basis of which, it could be concluded that further inquiry into such a deadly weapon of $\mathrm{M}$. phaseolina might be of significant value in the regulation of the diseases caused by this phytopahogen. The model of the enzyme can be effectively used to design mutagenesis experiments and broad screening on inhibitors of the protein could be done as well using knowledge obtained from this study. Last but not least, the study paves the way to asses $M$. phaseolina as a potential bioremedial agent such as in phenolic- rich effluent olive oil waste water.

\section{References:}

[1] Gold MH \& Alic M, Microbiological Reviews. 1993 57: 605 [PMID: 8246842]

[2] Doyle WA \& Smith AT, Biochem J. 1996 315: 15 [PMID: 8670100]

[3] Islam MS et al. BMC Genomics. 2012 13: 493 [PMID: 22992219]

[4] Sayadi S \& Ellouz R, J Chem Technol Biotechnol. 1993 57: 141

[5] Hamdi M et al. Appl Microbiol Biotechnol. 1991 34: 829

[6] Moreno E et al. Microbios. 1987 51: 169
[7] Sayadi S \& Ellouz R, Appl Environ Microbiol. 1995 61: 1098 [PMID: 16534959]

[8] Tien M \& Kirk TK, Proc Natl Acad Sci U S A. 1984 81: 2280 [PMID: 16593451]

[9] Reiser J et al. Appl Environ Microbiol. 1993 59: 2897 [PMID: 8215362]

[10] http://www.ncbi.nlm.nih.gov/

[11] http://web.expasy.org/protparam/

[12] http://web.expasy.org/peptidecutter/

[13] http://www.ncbi.nlm.nih.gov/Structure/cdd/wrpsb.cgi

[14] http://www.sbg.bio.ic.ac.uk/phyre2/html/page.cgi?id=in dex

[15] http://www.ebi.ac.uk/thorntonsrv/databases/pdbsum/Generate.html

[16] Morris AL et al. Proteins. 1992 12: 345 [PMID: 1579569]

[17] Dundas J et al. Nucl Acids Res. 2006 34: W116. [PMID: 16844972]

[18] Panda S \& Chandra G, Bioinformation. 2012 8: 891 [PMID: 23144546]

[19] http:/ / web.expasy.org/docs/expasy_tools05.pdf

[20] Stein L, Nat Rev Genet. 2001 2: 493 [PMID: 11433356]

[21] Ochoa A et al. BMC Bioinformatics. 2011 12: 90 [PMID: 21453511]

[22] Schwede $\mathrm{T}$ et al. Nucleic Acids Res. 2003 31: 3381[PMID: 12824332]

[23] Fidanova S \& Lirkov I, Seria Matematica-Informatica. 2009 17: 33

[24] Kelley LA \& Sternberg MJ, Nat Protoc. 2009 4: 363 [PMID: 19247286]

[25] Laurie AT \& Jackson RM, Curr Protein Pept Sci. 2006 7: 395 [PMID: 17073692]

[26] Huang B \& Schroeder M. BMC Structural Biology 2006 6:19 [PMID:16995956]

Edited by $P$ Kangueane

Citation: Akbar et al. Bioinformation 9(14): 730-735 (2013)

License statement: This is an open-access article, which permits unrestricted use, distribution, and reproduction in any medium, for non-commercial purposes, provided the original author and source are credited 


\section{Supplementary material:}

Table 1: Result from PeptideCutter

\begin{tabular}{|c|c|c|}
\hline Name of enzyme & $\begin{array}{l}\text { No. of } \\
\text { cleavages }\end{array}$ & Positions of cleavage site \\
\hline Arg-C proteinase & 13 & 3449507983106150157173230252301311 \\
\hline Asp-N endopeptidase & 25 & 2350546975768794107113123131158180191207210217224243249282288301309 \\
\hline $\begin{array}{l}\text { Asp-N endopeptidase }+\mathrm{N}- \\
\text { terminal Glu }\end{array}$ & 32 & $\begin{array}{l}23345054626975768794102107113123126131158165180189191207210217224241243 \\
249282288301309\end{array}$ \\
\hline BNPS-Skatole & 5 & 5767224257266 \\
\hline $\mathrm{CNBr}$ & 4 & 1117274277 \\
\hline Caspase1 & 1 & 250 \\
\hline $\begin{array}{l}\text { Chymotrypsin-high } \\
\text { specificity (C-term to } \\
\text { [FYW], not before P) }\end{array}$ & 22 & 395767688690104115126136183188206207224228229257260266270 \\
\hline $\begin{array}{l}\text { Chymotrypsin-low } \\
\text { specificity (C-term to } \\
\text { [FYWML], not before P) }\end{array}$ & 59 & $\begin{array}{l}1239101120223239434446485764676869848690100102104112115117119122126 \\
133136140147179183188193196200206207217224228229234241247251257260266270 \\
274277280287\end{array}$ \\
\hline Clostripain & 13 & 3449507983106150157173230252301311 \\
\hline Formic acid & 25 & 2451557076778895108114124132159181192208211218225244250283289302310 \\
\hline Glutamyl endopeptidase & 7 & 3563103127166190242 \\
\hline Iodosobenzoic acid & 5 & 5767224257266 \\
\hline LysC & 13 & 5335965118154186204212245276300304 \\
\hline LysN & 13 & 4325864117153185203211244275299303 \\
\hline $\begin{array}{l}\text { NTCB (2-nitro- } \\
\text { 5thiocyanobenzoic acid) }\end{array}$ & 6 & 52738893144289 \\
\hline Pepsin (pH1.3) & 92 & $\begin{array}{l}12238991010111920212231323839424343444546565763646668686984869099 \\
100103111112114115118121125126132133135136139140146169178179182183187192 \\
193195196205207216217223224227228228229233234240241246250251256257259260 \\
265266269270279280286287\end{array}$ \\
\hline Pepsin $(\mathrm{pH}>2)$ & 76 & $\begin{array}{l}12238991010111920212231323839424343444546636468686984869099100111 \\
112114115118121132133135136139140146169178179182183187192193195196205207 \\
216217227228233234240241246250251259260279280286287\end{array}$ \\
\hline Proteinase K & 157 & $\begin{array}{l}238910111314151719202225282931323536394344464756576061636467686973 \\
788081828486909899100101103104105110112113115116119120122123126127128130 \\
131133134136137139140141142143144147151152153155162165166169176178179180 \\
183184185188190191193194195196197199202203206207213217220223224226228229 \\
232233234236237239240241242247248249251253254255257258259260262265266267 \\
268269270271273278279280284287288291293294296297298303305306308309312\end{array}$ \\
\hline Name of enzymes & No.of cleavages & Positions of cleavage sites \\
\hline Staphylococcal peptidase I & 7 & 3563103127166190242 \\
\hline Thermolysin & 106 & $\begin{array}{l}12789101314161819212728303138424345465960676872798081838589979899 \\
100109111112115116118121122130133135136138139140142146150151152154164175 \\
177178179182183184187193194195196198202205206212216227233238240246247253 \\
254258259261264266267268270272273276278279286292293295296304307308311\end{array}$ \\
\hline Thrombin & 1 & 150 \\
\hline Trypsin & 26 & 53334495059657983106118150154157173186204212230245252276300301304311 \\
\hline
\end{tabular}

Table 2: Physiochemical characters as predicted by Expasy's prot-param program

\begin{tabular}{lll}
\hline Ramachandran plot statistics & No. of residues & Percentage \\
\hline Residues in the most favored region(A,B,L) & 235 & $83.9 \%$ \\
Residues in the additional allowed region $(\mathrm{a}, \mathrm{b}, 1, \mathrm{p})$ & 37 & $13.2 \%$ \\
Residues in the generously allowed region $(\mathrm{a}, \mathrm{b}, 1, \mathrm{p})$ & 3 & $1.1 \%$ \\
Residues in the disallowed region & 5 & $1.8 \%$ \\
Number of non-glycine \& non-proline residues & 280 & $100.0 \%$ \\
Number of end residues (excl. gly \& pro) & 2 & \\
Number of glycine residues (shown in triangles) & 20 & \\
Number of proline residues & 10 & \\
Total number of residues & 312 & \\
\hline
\end{tabular}

